\title{
Synthesis, Characterization and Unusual Dielectric Behaviour of $\lambda$-Shaped Mesogenic Homologous Series With Polar Substituents:
}

\author{
P. B. Chand ${ }^{1 *}$ and R. Manohar ${ }^{2}$ \\ ${ }^{1}$ Department of Physics, Siddhanath Science Campus, Mahendranagar, Kanchanpur, Nepal \\ ${ }^{2}$ Department of Physics, University of Lucknow, Lucknow-226007, India \\ purnachandmnr@hotmail.com
}

\begin{abstract}
A new $\lambda$-shaped mesogenic homologous series with polar bromo and nitro substituent was synthesized by fixing rigid 2-bromo-4nitrophenylazo groups to a resorcinol moiety. 4-n-alkoxybenzoyl groups esterify both the phenolic -OH groups. In the present series, methoxy and ethoxy derivatives are non-mesogenic, n-propyloxy to n-octyloxy derivatives exhibit enantiotropic nematic mesophase. Smectic C (SmC) mesophase commences from the $n$ - octyloxy derivative as an enantiotropic phase and is retained up to the last member synthesized. The effect of polar bromo and nitro substituent on mesomorphic properties is discussed. In this paper, we also report the unusual dielectric behaviour of n-octyloxy and $n$-tetradecyloxy derivatives of the present $\lambda$-shaped mesogenic homologous series. The dielectric permittivity of the sample was found to be unusually low in SmC phase in comparison to nematic phase. We have explained it on the basis of two-conformer model, which can exist in SmC phase due to specific shape of the molecule. The model has been supported by calculating activation energy of both the possible conformers from Arrhenius plot of relaxation frequency versus temperature. There is a clear difference in the value of activation energy of both the conformers as expected.
\end{abstract}

Keywords: C. Differential scanning calorimetry (DSC); D. Dielectric properties; D. Phase transitions

\section{Introduction}

Liquid crystalline materials are of great interest for material science as well as for life sciences. Their properties can be tuned by appropriate molecule design i.e. a calamatic structure or a discotic one. The design of novel thermotropic liquid crystals as advanced functional materials involves suitable selection of a core fragment, linking group and terminal functionality. In most cases, a significant mesophase destabilization is connected with this structural variation; however in rare cases lateral substituents can have a mesophase stabilizing effect.

Lateral substitution at the centre of the mesogen increases the molar volume and decreases the LC packing density [1], thereby hindering their ability to form any kind of liquid crystalline phase if the mesogen is short [2], and smectic mesophases if the mesogen is more extended $[2,3]$.

Recently molecular topology [4] has attracted much attention for producing molecules of different shapes with mesogenic behaviour.
Recent review of research work shows that a number of efforts have been going on for designing molecules of different shape showing liquid crystalline behaviour. Reports in the literature have revealed that the majority ( $90 \%$ ) of the published work on bent, banana or V-shaped, based on rigid central 1, 3-phenylene and 2, 7-naphthylene cores self-assemble to yield molecular structures that exhibit banana liquid crystalline polymorphism [5]. The swallowed tailed compounds [6] have also been reported with interesting liquid crystalline phases. "Janus-like" super molecular liquid crystals have been found with chiral nematic and chiral smectic phases [7]. Yoshizawa and Yamaguchi [8] have reported layered structure in the nematic phase consisting of $U$ shaped molecule. Yamaguchi et al. [9] have reported synthesis of homologous series of novel $\lambda$-shaped mesogenic compounds, which showed unusual smectic phases. They have investigated the physical properties of $\lambda$-shaped mesogenic compounds by means of DSC, XRD and optical microscopy. Yoshizawa et al. [10] have designed novel Y-shaped liquid crystal oligomers in which three mesogenic units are connected via 3, 5-dihydroxybenzoic acid. The Y-shaped liquid 
crystal oligomers were found to show a direct phase transition from isotropic liquid to anticlinic $\mathrm{SmC}$ (SmCanti) phase. They observed that the Y-shaped compounds are quite different from that of the corresponding $\lambda$-shaped mesogenic compounds. Earlier we [11] have reported the synthesis of two $\lambda$ shape mesogenic homologous series of in which one series having methyl and other having ethoxy terminal substituents. Mahajan et al. [12] have also reported synthesis of two $\lambda$ shape mesogenic homologous series of 1, 2, 4-trisubstituted benzene derivative possessing ester and amide linkages having terminal chloro or methoxy substituents.

In the present work, we report the synthesis of a new $\lambda$-shaped mesogenic homologous series of azoester containing polar bromo and nitro substituents to evaluate the effect of polar substituents on mesomorphism. We also report the dielectric characterization of two representative members, $n$-octyloxy and $n$-tetradecyloxy derivatives of this newly synthesized $\lambda$-shaped homologous series. The $\lambda$ shape molecular topology is expected to produce a characteristic effects since two different non-symmetric molecular configuration co-exist in a single molecule. The dielectric response of planar aligned sample is expected to be the result of more than one relaxation process because of their particular molecular shape, therefore the Cole-Cole presentation have been used to study the molecular relaxation process for both the representative compounds.

\section{Experimental details}

\subsection{Characterization}

Microanalyses of the compounds were carried out on a Coleman carbon-hydrogen analyzer and the values obtained are in close agreement with those calculated. IR spectra were determined as $\mathrm{KBr}$ pellets, using a Shimadzu IR-8400 spectrophotometer. ${ }^{1} \mathrm{H}$ NMR spectra were obtained with a Perkin-Elmer R-32 spectrometer using tetramethylsilane (TMS) as the internal reference standard. The chemical shifts are quoted as $\delta$ (parts per million) downfield from the reference. $\mathrm{CDCl}_{3}$ was used as a solvent for all the compounds. Liquid crystalline properties were investigated on a Leitz Laborlux 12 POL microscope provided with a heating stage. The enthalpies of transitions, reported as $\mathrm{J} \mathrm{g}^{-1}$, were measured on a Mettler TA-4000 system, at a scanning rate of $5^{\circ} \mathrm{C} \mathrm{min}^{-1}$. The instrument was calibrated using pure indium as a standard.

4-Hydroxybenzoic acid, the appropriate $n$-alkyl bromides (BDH), $\mathrm{KOH}$, resorcinol, bromine, 4-nitroaniline, $\mathrm{NaNO}_{2}$, thionyl chloride etc. (Sisco Chem.) were used as received. All the solvents were dried and distilled prior to use.

Compounds of the new series I was prepared following the pathway shown in Fig. 1.

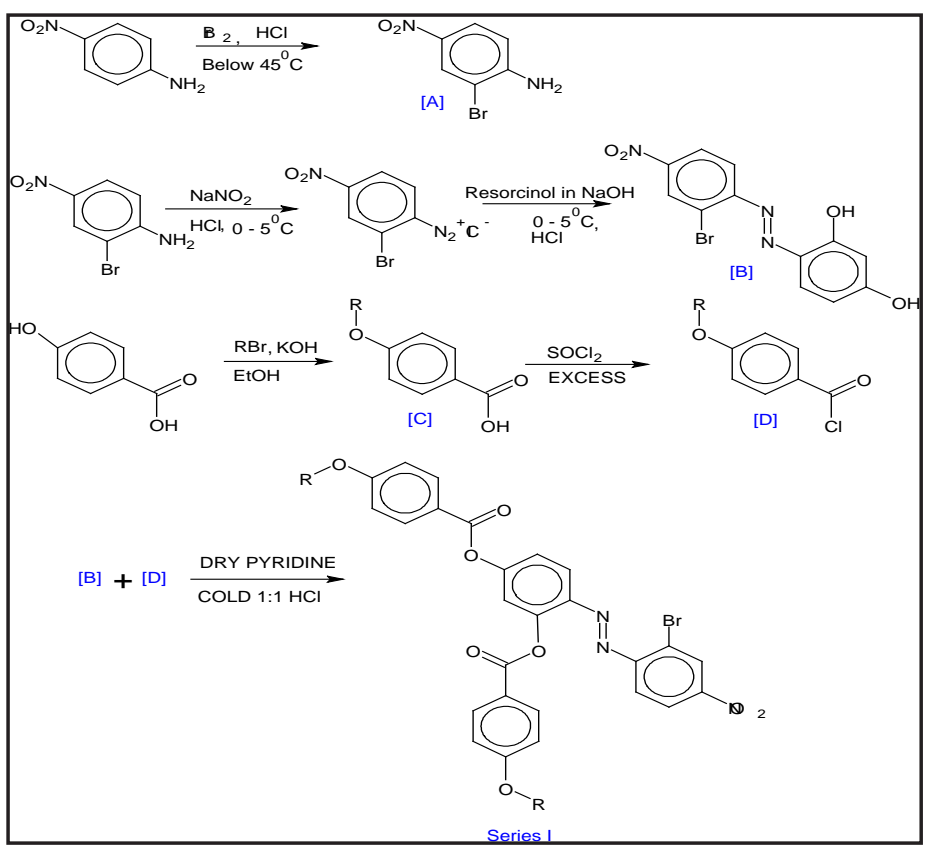

Fig. 1. Synthetic route to series I.

\subsubsection{2-Bromo-4-nitroaniline [A]}

This was prepared from 4-nitroaniline by the reported method [13].

\subsubsection{Bromo-4-nitro 2', 4'-dihydroxy azobenzene [B]}

The compound B was synthesized by using the diazotization of compound A and coupling it with resorcinol [14]. The crude dye was crystallized repeatedly from ethanol till a constant melting point was obtained. m. p.: $197^{\circ} \mathrm{C}$. Elemental analysis: found $\mathrm{C}$ 42.13, $\mathrm{H} 2.08, \mathrm{~N} 12.61 \%, \mathrm{C}_{12} \mathrm{H}_{8} \mathrm{~N}_{3} \mathrm{O}_{4} \mathrm{Br}$ requires $\mathrm{C} 42.06, \mathrm{H} 2.37$, and N $12.43 \%$. The IR spectrum of the compound showed a broad peak of intermolecular hydrogen bonded phenolic $-\mathrm{OH}$ between 
3500 and $3300 \mathrm{~cm}^{-1}$. Other signals observed were at 2921, 1610 $(-\mathrm{N}=\mathrm{N}-), 1589,1502,1475,1406,1305,1273,1247,1192,1120$, 1035, 877, 839, 658, $565(\mathrm{Br}) \mathrm{cm}^{-1}$.

\subsubsection{4-n-Alkoxybenzoic acids [C] and 4-n- alkoxybenzoyl chlorides [D]}

These compounds were synthesized by a modified method of Dave and Vora [15].

\subsubsection{Bromo-4-nitro-2', 4'-bis alkoxybenzoyloxy) azobenzenes [Series I]}

(4-n-

Compound B (0.02 mol) was dissolved in dry pyridine $(5 \mathrm{ml})$ and a cold solution of Compound D (0.04 mol) in dry pyridine $(5 \mathrm{ml})$ was added slowly to it in an ice bath with constant stirring. The mixture was allowed to stand overnight at room temperature. It was acidified with cold 1: 1 aqueous hydrochloric acid. The solid was separated, dried and triturated by stirring for 30 min with $10 \%$ aqueous sodium hydroxide solution and then washed with water. The insoluble product was thus separated from the reactants and was chromatographed on silica gel using petroleum ether $\left(60-80^{\circ} \mathrm{C}\right)$-ethyl acetate mixture (96:4) as eluent. Removal of solvent from the elute afforded as solid material which was crystallized repeatedly from ethanol till constant transition temperatures were obtained. The purities of all these compounds were checked by thin layer chromatography. Elemental analyses of all the compounds of series I was found to be satisfactory. IR and ${ }^{1} \mathrm{H}$ NMR spectral data of n-octyloxy derivative are given below.

IR spectrum $\left(v_{\max } / \mathrm{cm}^{-1}\right): 2921,1685$ (-COO-), 1605 $(-\mathrm{N}=\mathrm{N}-), 1580,1511,1500,1467,1430,1307,1255,1170$, 1124, 1016, 941, 843, 773, 647, 550. ${ }^{1} \mathrm{H}$ NMR spectrum: $\delta 0.9\left(\mathrm{t}, 6 \mathrm{H}, 2 \mathrm{x}-\mathrm{CH}_{3}\right), 1.25-1.50\left(\mathrm{~m}, 20 \mathrm{H}, 10 \mathrm{x}-\mathrm{CH}_{2}^{-}\right)$, 1.79 (qunt. 4H, 2 x - O-C- $\mathrm{CH}_{2}^{-}$), 4.00 (t, 4H, $2 \times \mathrm{ArOCH}_{2}^{-}$), $6.90(\mathrm{~d}, \mathrm{~J}=9 \mathrm{~Hz}, 4 \mathrm{H}, \mathrm{ArH}), 7.03(\mathrm{~d}, \mathrm{~J}=9 \mathrm{~Hz}, 2 \mathrm{H}, \operatorname{ArH}), 7.96$ (d, J=9Hz, 4H, ArH), 8.50(s, 1H, ArH), 8.70 (d, 2H, ArH), $9.00(\mathrm{~s}, 1 \mathrm{H}, \mathrm{ArH})$.

\subsection{Preparation of cell and measurement of dielectric parameters}

Two similar cells have been prepared by using highly conducting ITO coated optically flat glass substrates, which were used as electrodes. These electrodes allow us to align the liquid crystal molecules. To get planar alignment all the electrodes were pretreated with adhesion promoter and polymer (Nylon 6/6), and then rubbed unidirectionally with velvet cloth. The thickness of the cell was maintained at $5 \mu \mathrm{m}$ by means of Mylar spacer. The homeotropically-aligned cells for measurement of parallel component of dielectric permittivity, were prepared by using lecithin as aligning material. The cells have been calibrated using standard AR grade liquids like Benzene and $\mathrm{CCl}_{4}$. The materials were introduced into the cell by capillary action at $5^{\circ} \mathrm{C}$ higher than their isotropic transition temperature, and then cooled slowly in the presence of a small electric field for obtaining well-aligned cells. Their alignment was conformed by observing them with polarizing microscope model CENSICO (7626).

The dielectric behaviour of materials has been studied in the frequency range $100 \mathrm{~Hz}-10 \mathrm{MHz}$ using a computer controlled Impedance/gain phase analyzer Hewlett/Packard (HP4194A). Measurements in the higher frequency range have been limited to $10 \mathrm{MHz}$ because of dominating effect of ITO coated on the glass plates [16]. The dielectric parameters were measured as a function of temperature, by keeping the sample on a hot stage of computer controlled refrigerator circulator (Julabo F25). The experiments were done at very slow heating rate and temperature was measured with the accuracy of $\pm 0.01^{\circ} \mathrm{C}$.

\section{Results and Discussion}

\subsection{Optical Microscopic Studies}

As a preliminary investigation, the mesophases exhibited by series I was examined using a polarizing optical microscope. Thin film of the samples was obtained by sandwiching them between a glass slide and a cover slip. First two members synthesized $(n=1$ \& 2 ) of the series I is non-mesogenic. On cooling from the isotropic liquid in an ordinary slide, $n$-propyloxy to $n$-octyloxy derivatives of the series I show the threaded/marble texture characteristic of nematic mesophase (Fig. 2a). On further cooling, only the $n$-octyloxy derivative show schlieren texture characteristic of SmC mesophase (Fig. 2b). On cooling from the isotropic liquid, the $n$-decyloxy to $n$-hexadecyloxy derivatives shows schlieren texture characteristic of SmC mesophase. 


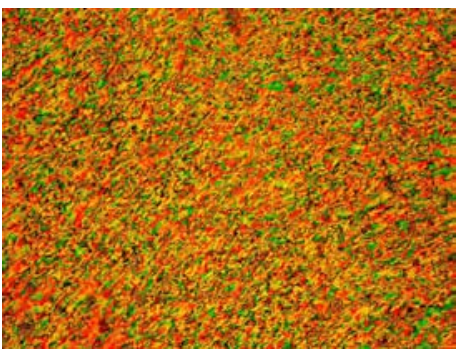

(a)

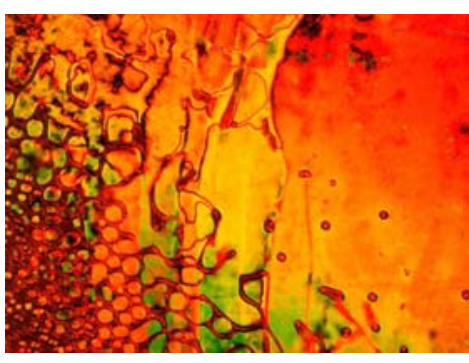

(b)
Fig. 2. Optical textures of (a) Compound $8\left(130^{\circ} \mathrm{C}\right)$ (b) Compound $8\left(115^{\circ} \mathrm{C}\right)$

\subsection{DSC Studies}

As representative cases, the associated enthalpies of transition of the $n$-octyloxy and the $n$-tetradecyloxy derivatives of series I were measured by differential scanning calorimetric (DSC). Data are recorded in Table 1. Enthalpy changes of the various transitions agree well with the existing related literature value [17].

Table 1. DSC data for series I compound.

\begin{tabular}{|c|c|c|c|c|}
\hline Derivative & Transition & $\begin{array}{c}\text { Peak Temp. } \\
\left({ }^{\circ} \mathrm{C}\right)\end{array}$ & $\Delta \mathrm{H}(\mathrm{J} / \mathrm{g})$ & $\Delta \mathrm{S}\left(\mathrm{J} / \mathrm{g}^{\circ} \mathrm{K}\right)$ \\
\hline \multirow[t]{3}{*}{ n-octylosxy } & Cr-SmC & 88.500 & ${ }^{\otimes} 78.120$ & 0.216 \\
\hline & SmC-N & 121.000 & 6.170 & 0.016 \\
\hline & N-I & 130.000 & 0.980 & 0.002 \\
\hline \multirow[t]{2}{*}{$n$-tetradecyloxy } & Cr-SmC & 87.500 & ${ }^{\otimes} 76.430$ & 0.212 \\
\hline & SmC-I & 131.000 & 1.770 & 0.004 \\
\hline
\end{tabular}

$\otimes$ : total enthalpy including any other $\mathrm{Cr}-\mathrm{Cr}$ transition

\subsection{The Phase Behaviour}

Twelve compounds were synthesized, and their mesogenic properties were evaluated. Methoxy and ethoxy derivatives are non-mesogenic, n-propyloxy to n-octyloxy derivatives exhibit enantiotropic nematic mesophase. Smectic C mesophase commences from the $n$ - octyloxy derivative as an enantiotropic phase and is retained up to the last member synthesized. The transition temperatures are recorded in Table 2. A plot of transition temperatures against the number of carbon atoms in the alkoxy chain (Fig. 3) shows smooth falling tendencies with pronounces odd-even effect for the $\mathrm{N}-\mathrm{I}$ transition temperatures with increasing chain length. The SmC - I transition temperatures also shows falling tendency as the series ascended.
Table 2. Transition temperatures $\left({ }^{\circ} \mathrm{C}\right)$ of the series I compound.

\begin{tabular}{|c|c|c|c|c|c|c|c|c|}
\hline $\begin{array}{c}\text { Compound } \\
\text { No. }\end{array}$ & $\begin{array}{c}\mathrm{R}=-\mathrm{C}_{\mathrm{n}} \mathrm{H}_{2 \mathrm{n}+1} \\
\mathrm{n}=\end{array}$ & $\mathrm{Cr}$ & & $\mathrm{SmC}$ & & $\mathrm{N}$ & & I \\
\hline 1 & 1 & $\bullet$ & 185 & - & - & - & - & • \\
\hline 2 & 2 & $\bullet$ & 170 & - & - & - & - & • \\
\hline 3 & 3 & $\bullet$ & 148 & - & - & $\bullet$ & 153 & • \\
\hline 4 & 4 & $\bullet$ & 143 & - & - & $\bullet$ & 166 & • \\
\hline 5 & 5 & $\bullet$ & 108 & - & - & $\bullet$ & 127 & • \\
\hline 6 & 6 & $\bullet$ & 103 & - & - & $\bullet$ & 139 & $\bullet$ \\
\hline 7 & 7 & $\bullet$ & 85 & - & - & $\bullet$ & 131 & • \\
\hline 8 & 8 & $\bullet$ & 88 & $\bullet$ & 122 & $\bullet$ & 131 & • \\
\hline 9 & 10 & • & 95 & • & 146 & - & - & • \\
\hline 10 & 12 & • & 90 & • & 140 & - & - & • \\
\hline 11 & 14 & • & 88 & • & 132 & - & - & • \\
\hline 12 & 16 & • & 95 & • & 131 & - & - & • \\
\hline
\end{tabular}

$\mathrm{Cr}=$ crystalline solid phase; $\mathrm{SmC}=$ smectic $\mathrm{C}$ phase;

$\mathrm{N}=$ nematic phase $\mathrm{I}=$ isotropic liquid phase

- = phase exists; - = phase does not exist

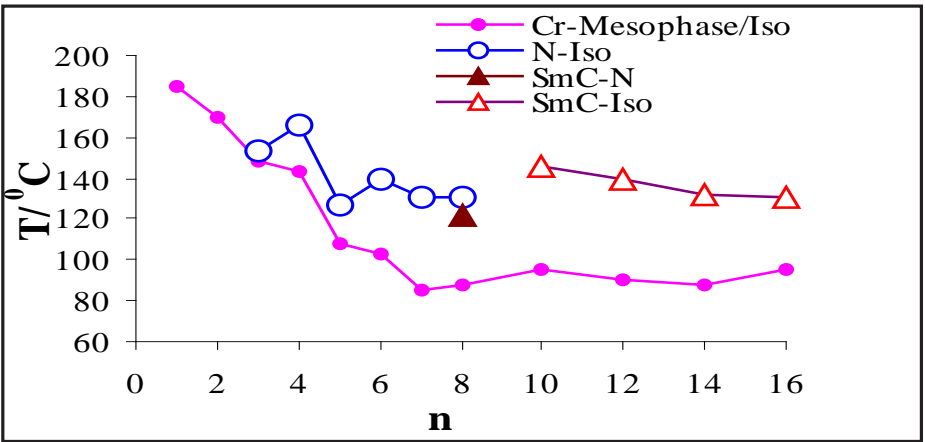

Fig. 3. The phase behaviour of series

\subsection{Mesogenic Properties and Molecular Constitution}

Fig. 4 shows the molecular shapes of the $n$-octyloxy derivative of the present series I in the respective all-trans conformation (energyminimized models derived from CS ChemDraw Ultra 7.0), from which it can be noticed that the molecules are neither rod-like nor disc-like.

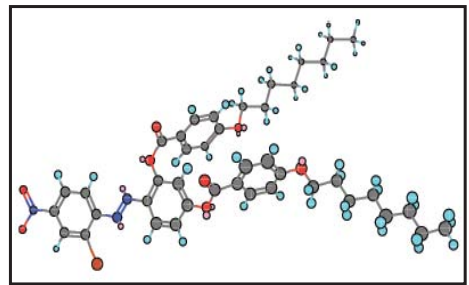

(a)

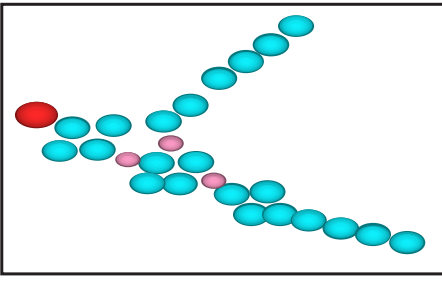

(b)
Fig. 4. Energy-minimized (a) Ball and stick model and (b) Space filling model (MM2 models derived from CS Chem draw Ultra 7.0 software) structures of n-octyloxy derivative 


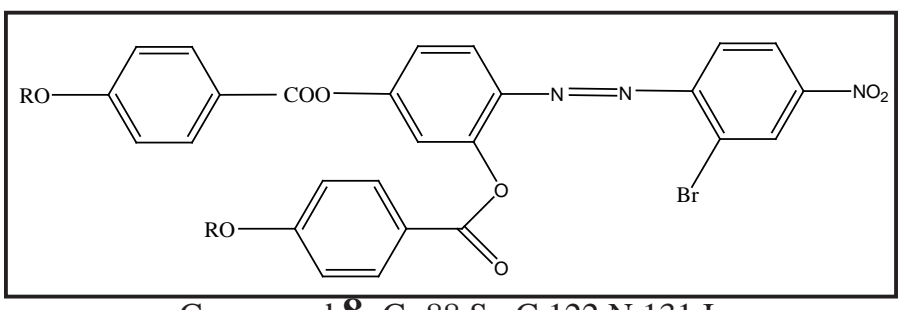

Compound 8. Cr 88 SmC $122 \mathrm{~N} 131$ Iso
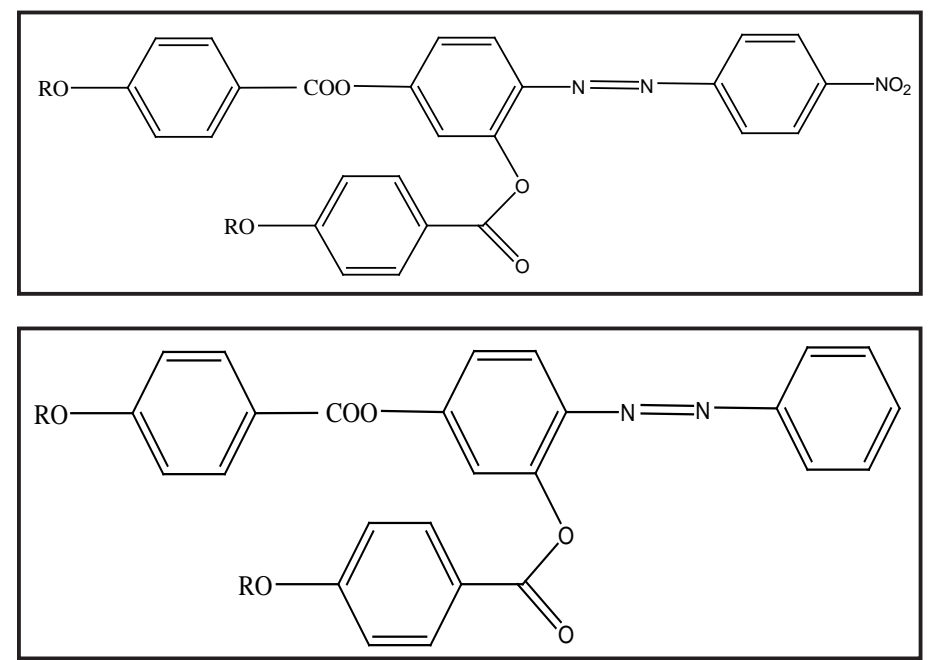

Compound A. Cr 71 SmA 116 Iso

Compound B. Cr (88 N) 101 Iso

$$
\mathrm{R}=-\mathrm{C}_{\mathrm{n}} \mathrm{H}_{2 \mathrm{n}+1} ; \mathrm{n}=8
$$

Fig. 5. The transition temperatures and molecular structures of representative compounds $\mathbf{8}$ of the present series I with other structurally related compounds $\mathbf{A}$ and $\mathbf{B}$.

Simply considering the length to breadth ratio of these molecules does not provide a convincing explanation of the occurrence of anisotropic liquid phases. It is well known that thermo tropic liquid crystals are highly sensitive to the molecular constitution. It is of prime importance from the chemist's point of view to find the effect of alteration in molecular core to the mesogenic properties of the compound. The thermal stability and mesophase length as a measure of mesomorphism can be correlated with the molecular constitution of the compounds. Fig. 5 shows the comparison of transition temperatures and molecular structures of representative compounds $8(n=8)$ of the present series I with other structurally related compounds A [18] and B [19] reported in the literature. The width of the smectic phase of present compound 8 is $34^{\circ} \mathrm{C}$ whereas that of compound $\mathrm{A}$ is $45^{\circ} \mathrm{C}$. Gray [20] has explained that the compound, which required more thermal energy to disorganize the molecular arrangement of the smectic mesophase, is thermally more stable. As can be seen in Fig. 5, more thermal energy has to be supplied to disorganize the molecular arrangement of the smectic melt of compound 8 , evidenced by the fact that the $\mathrm{SmC}$ $-\mathrm{N}$ transition temperature is higher by $6^{\circ} \mathrm{C}$ than that for the $\mathrm{SmA}$ - Iso transition temperature of compound $\mathrm{A}$. The higher smecticnematic transition temperatures of compound 8 may be due to the polar nature of the lateral bromo group which increases the overall polarizability of the molecules of compound 8 compared to compound A. The range and thermal stability of the mesophase is a more important factor in relating mesomorphic behaviour to chemical constitution since the chemical grouping gives rise to intermolecular attractions, which in turn determine the mesophase range and thermal stability. One should remember too that the length of the mesophase is determined partly by the $\mathrm{Cr}-\mathrm{Sm}$ phase transition temperatures.

Reference to Fig. 5 indicates that the thermal stability of nematic mesophase of compound 8 is higher by $43^{\circ} \mathrm{C}$, compared to compound $\mathrm{B}$. This is understandable, as the molecules of compound 8 are more polarizable compared to the molecules of compound $\mathrm{B}$ due to the presence of highly polar nitro and bromo substituents. The entire mesogenic homologous series of compound B is purely nematogenic whereas the presence of polar substituents in the molecule of present mesogenic homologous series induces the smectogenic tendencies.

\subsection{Dielectric behaviour}

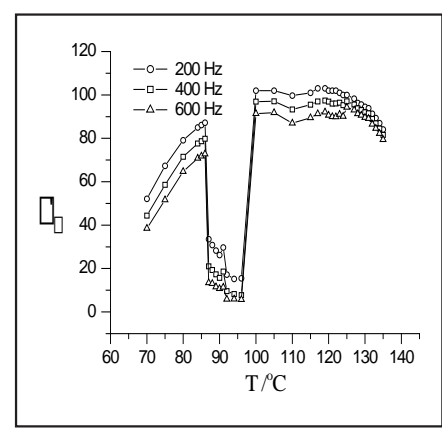

(a)

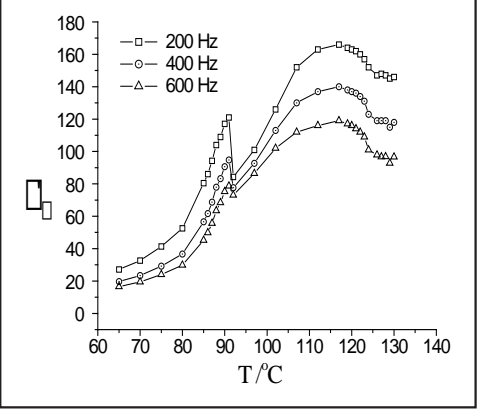

(b)
Fig. 6. Temperature dependence of the dielectric permittivity of (a) n-octyloxy derivative (b) and n-tetradecyloxy derivative. 


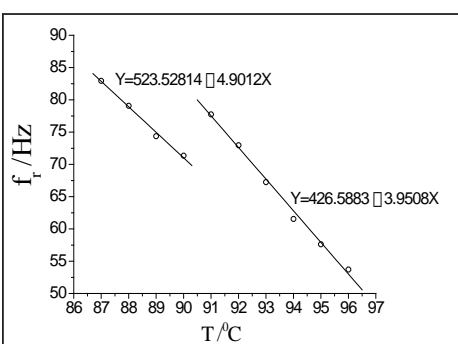

(a)

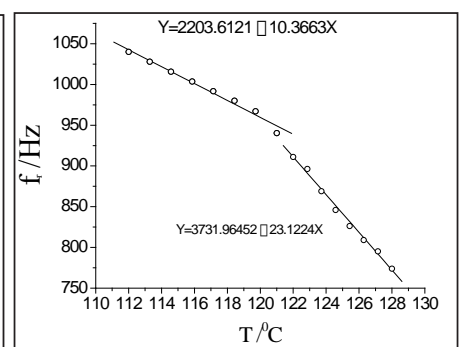

(b)
Fig. 7. The plot of relaxation frequency with temperature for (a) $n$-octyloxy derivative and (b) n-tetradecyloxy derivative in SmC phase.

The temperature dependence of the perpendicular component of dielectric permittivity for both n-octyloxy and n-tetradecyloxy derivatives of the present $\lambda$-shaped mesogenic homologous series is given in Fig. 6. It is clear from Fig. 6a that dielectric permittivity for n-octyloxy derivative increases with increase in temperature and shows a sharp fall near the $\mathrm{Cr}$ to $\mathrm{SmC}$ phase transition temperature i.e. $88^{\circ} \mathrm{C}$. A sharp discontinuity is again observed near the $\mathrm{SmC}$ to $\mathrm{N}$ phase transition at $122^{\circ} \mathrm{C}$ and dielectric permittivity is almost independent of temperature in $\mathrm{N}$ phase. Further increase in temperature results in the $\mathrm{N}$ to isotropic transition and after this transition temperature the liquid crystal sample behaves like any ordinary organic liquid. Similar type of behaviour is also observed by many researchers for liquid crystals molecules of different shape, but the magnitude of dielectric permittivity is much higher for present $\lambda$ shape sample in comparison to liquid crystal materials with molecules of other shapes. This may be due to the unique shape of molecules taken for the present work. The plot in Fig. 6 (a) clearly shows an unexpected discontinuity in SmC phase near $92^{\circ} \mathrm{C}$, which suggests the existence of temperature dependent dual molecular arrangement in the SmC phase of these $\lambda$ shaped molecules. The texture studies confirm that no reorientation of director is taking place. We have designated the SmC phase before $92^{\circ} \mathrm{C}$ as $\mathrm{SmC}_{1}$ and after $92^{\circ} \mathrm{C}$ as $\mathrm{SmC}_{2}$ phase. This type of molecular behaviour is also supported by the relaxation frequency versus temperature curve (discussed later) of the sample in smectic $\mathrm{C}$ phase (Fig. 7a). The existence of more than one molecular arrangement in any particular liquid crystalline phase has also been reported by other researchers [21].
Table 3. Variation of dielectric strength and distribution parameter in $\mathrm{SmC}$ phase of both n-octyloxy and n-tetradecyloxy derivatives.

\begin{tabular}{|c|c|c|c|}
\hline $\begin{array}{c}\text { Temp } \\
\text { erature } \\
\left({ }^{\circ} \mathrm{C}\right)\end{array}$ & $\begin{array}{l}\text { Dielectric strengthn- } \\
\text { octyloxy derivative }\end{array}$ & $\begin{array}{l}\text { Distribution } \\
\text { parameter }\end{array}$ & $\begin{array}{c}\text { Dielectric } \\
\text { Anisotropy }\end{array}$ \\
\hline 90 & 105.571 & 0.044 & -34.345 \\
\hline 91 & 104.785 & 0.037 & -34.849 \\
\hline 92 & 104.906 & 0.033 & -23.376 \\
\hline 94 & 104.333 & 0.016 & -30.200 \\
\hline 96 & 104.685 & 0.020 & -34.459 \\
\hline 100 & 89.978 & 0.107 & -35.651 \\
\hline 105 & 95.280 & 0.114 & -34.208 \\
\hline 110 & 100.713 & 0.087 & -33.000 \\
\hline 115 & 103.348 & 0.083 & -31.826 \\
\hline 117 & 102.327 & 0.092 & -32.300 \\
\hline 119 & 101.005 & 0.092 & -29.422 \\
\hline 120 & 108.221 & 0.097 & -31.300 \\
\hline 121 & 108.215 & 0.102 & -27.900 \\
\hline 122 & 107.957 & 0.105 & -24.933 \\
\hline 123 & 106.821 & 0.097 & -22.735 \\
\hline 124 & 105.435 & 0.103 & -19.689 \\
\hline 125 & 100.281 & 0.117 & -17.971 \\
\hline 127 & 98.017 & 0.134 & -10.231 \\
\hline 128 & 96.483 & 0.156 & -4.231 \\
\hline
\end{tabular}

n-tetradecyloxy derivative

\begin{tabular}{llll}
\hline 107 & 139.015 & 0.221 & -22.901 \\
112 & 161.895 & 0.154 & -55.168 \\
117 & 170.143 & 0.154 & -63.275 \\
119 & 168.163 & 0.148 & -58.397 \\
120 & 160.682 & 0.130 & -55.832 \\
121 & 160.437 & 0.116 & -52.053 \\
122 & 156.178 & 0.091 & -46.374 \\
123 & 149.249 & 0.058 & -36.985 \\
\hline
\end{tabular}


Vol. II

\begin{tabular}{llll}
\hline 124 & 143.834 & 0.044 & -20.702 \\
126 & 133.809 & 0.001 & -7.763 \\
127 & 135.952 & 0.001 & -10.901 \\
128 & 133.809 & 0.001 & -6.595 \\
\hline & 131.675 & 0.012 & \\
\hline
\end{tabular}

Fig. 8. (a) Represent the topological concept of the $\lambda$ shape molecule,

(b) is showing the two different possible conformers in $\lambda$-shaped molecule liquid crystal samples. And the arrows are showing the most preferred alignment direction of the molecule. The induced polarization sign for these conformers will be opposite.

Usually the dielectric permittivity in smectic phase is higher than dielectric permittivity in nematic phase, while in the present work the dielectric permittivity in smectic $\mathrm{C}$ phase is lower than the dielectric permittivity in nematic phase.This is due to the negative dielectric anisotropy of the samples. The values of dielectric anisotropy as determined by parallel and perpendicular component of dielectric permittivity has been given in table 3. This unusual behaviour can be explained by a model of two different molecular conformers of oppositely induced dipole moment. The $\lambda$ shape molecular topology suggests the coexistence of two different non symmetric arms in a single molecule (Fig. 8a). The behaviour may be explained by a model of dynamically fluctuating two interconvertible conformers separated by a temperature dependent energy barrier. The two possible conformers of $\lambda$ shape molecules are as presented in Fig. 8b. It is clear from the figure that both of these molecule conformers have oppositely induced dipole moment.

Let the $\mathrm{n}_{1}, \mathrm{n}_{2}$ and $\mathrm{p}_{1}, \mathrm{p}_{2}$ are the concentration and induced polarization of species one and two respectively and the concentration of these two species is dependent on temperature. Therefore when the induced dipole moments of these two conformers counteract, the net induced polarization will vanishes or attain a minimum value depending on their respective concentration i.e. $n_{1} p_{1}=n_{2} p_{2}[22,23]$. This may be the reason behind the exceptionally low values of dielectric permittivity in $\mathrm{SmC}$ phase of such sample.

The variation of dielectric permittivity with temperature for second derivative of the series i.e. $n$-tetradecyloxy derivative is shown in Fig. 6b. The nature of dielectric permittivity in crystalline phase is almost similar to that observed for the previous element. After showing a discontinuity near $\mathrm{Cr}$ to $\mathrm{SmC}$ phase transition temperature i.e. $88^{\circ} \mathrm{C}$, dielectric permittivity increases gradually till isotropic transition temperature because nematic phase does not exist. The dual molecular arrangement in the sample is not as clear from the Fig. $6 \mathrm{~b}$ as for the first sample. But existence of dual molecular arrangement can be verified from Fig. $7 \mathrm{~b}$ i.e. relaxation frequency versus temperature curve, as the whole plot can be divided into two parts (discussed later) in SmC phase.

The dielectric relaxation phenomenon of such a typical molecular structure must be due to the combined effect of more than one relaxation phenomenon. To ascertain the existence of dynamically fluctuating two interconvertible conformers separated by a temperature dependent energy barrier we have determined the relaxation frequency of the samples using the Cole-Cole presentation. The Cole -Cole dispersion equation may be given as [24, 25]

$$
\varepsilon^{\prime \prime}=\varepsilon^{\prime}(\infty)+\frac{\delta \varepsilon^{\prime}}{1+(j \omega \tau)^{(1-\alpha)}} \ldots \ldots \ldots .
$$

where $\delta \varepsilon^{\prime}$ is dielectric strength of material, $\varepsilon^{\prime}(\infty)$ is high frequency of dielectric permittivity of free space, $\omega$ is the angular frequency, $\tau$ is relaxation time and $\alpha$ is distribution parameter. If the value of $\alpha$ is small the above equation will satisfy the Debye type of relaxation phenomenon but for $\alpha$ value more than 0.5 the 
above equation suggests the existence of more than one relaxation process [26].

The errors of experimental data in low and high frequency range due to such type of sample holder are quite common.[16, 27] therefore the experimental results extremely required a low and high frequency correction. On separating real and imaginary part of the equation (1) one may get after adding high and low frequency correction parameters

$\varepsilon^{\prime}=\varepsilon^{\prime}(d c) f^{-n}+\varepsilon^{\prime}(\infty)+\frac{\delta \varepsilon^{\prime}\left[1+(2 \pi f \tau)^{(1-\alpha)} \sin (\alpha \pi / 2)\right]}{1+(2 \pi f \tau)^{2(1-\alpha)}+2(2 \pi f \tau)^{(1-\alpha)} \sin (\alpha \pi / 2)} \cdots$

and

$$
\varepsilon^{\prime \prime}=\frac{\sigma(d c)}{\varepsilon_{0} 2 \pi f^{k}}+\frac{\delta \varepsilon^{\prime}(f \tau)^{(1-\alpha)} \cos (\alpha \pi / 2)}{1+(2 \pi f \tau)^{2(1-\alpha)}+2(2 \pi f \tau)^{(1-\alpha)} \sin (\alpha \pi / 2)}+A f^{m} \ldots
$$

where $\sigma(d c)$ is ionic conductance and $\varepsilon_{o}$ is free space permittivity, $f$ is frequency, while $n, m$ and $k$ are fitting parameters. The term $\varepsilon^{\prime}$ (dc) $f^{-n}$ and $\sigma(d c) / \varepsilon_{o} 2 \pi f^{k}$ are added in above equations for low frequency effect due to the electrode polarization, capacitance and ionic conductance. The term $A f^{m}$ is added in equation 3 for high frequency effect due to the ITO resistance and lead inductance. By the least square fitting of above equation into experimental data we have removed the low and high frequency errors.

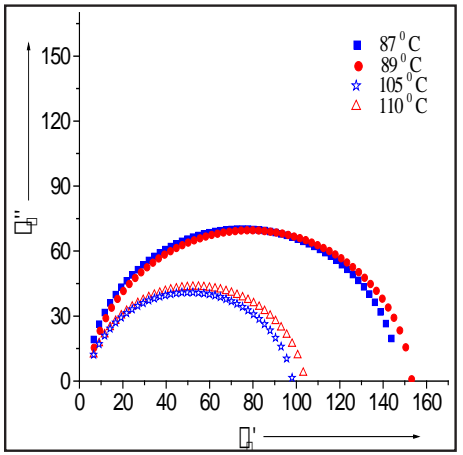

(a)

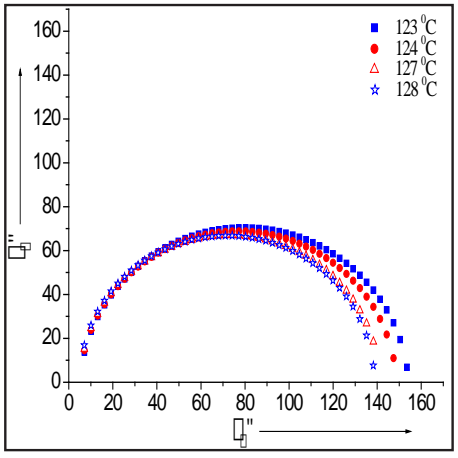

(b)
Figure 9: The Cole-Cole plot for (a) n-octyloxy derivative and (b) n-tetradecyloxy derivative in SmC phase. The solid legends represents the $\mathrm{SmC}_{1}$ phase and open legend represents the $\mathrm{SmC}_{2}$ Phase in different samples.

The Cole-Cole plot of first $n$-octyloxy derivative of the series in the $\mathrm{SmC}$ phase is shown in Fig. 9a. Using such plots dielectric parameters such as distribution parameter; relaxation frequency and dielectric strength have been evaluated. The plot between the relaxation frequency and temperature for $n$-octyloxy derivative in SmC phase has been shown in Fig. 7a.

It is clear from Fig. 7a that the whole temperatures range of $\mathrm{SmC}$ phase can be divided into two parts, shown by two different straight lines of different slopes. The activation parameter has been calculated using $\tau=\tau_{0} \exp \left(\mathrm{W}_{\mathrm{b}} / \mathrm{kT}\right)$----- (4) where $W_{b}$ is activation energy, $k$ is Boltzmann constant and $\tau$ is relaxation time.

The activation barrier in $\mathrm{SmC}$ phase towards lower temperature side is $3.56 \mathrm{~kJ} / \mathrm{mol}$, where as towards higher temperature side it was $5.8 \mathrm{~kJ} / \mathrm{mol}$. Which confirms the existence of two interconvertible conformers separated by a temperature dependent energy barrier and support the observation made by dielectric permittivity curve. Previously Dhar et al. [27] and Ratna et al. [28] have also reported the existence of two SmA phase on the basis of two different activation energies in the particular temperature range.

Similar type of behaviour of relaxation frequency is also observed for $n$-tetradecyloxy derivative in smectic $\mathrm{C}$ phase (Fig. 7b). Here the two energies are found to be $1.44 \mathrm{~kJ} / \mathrm{mol}$ and $4.44 \mathrm{~kJ} / \mathrm{mol}$. The relaxation frequency again shifts towards the lower frequency side at higher temperature and the figure shows the existence of more than one molecular arrangement in smectic $C$ phase. Such a typical behaviour of relaxation frequency for n-tetradecyloxy derivative of this series conforms that increase in temperature produces the distortion in shape of molecule, which is also supported by Table 3 . The dielectric strength of the sample decreases drastically at higher temperature. Similar type of behaviour of relaxation frequency is also reported by our group earlier and the relaxation process may be correlated with the rotation of side chain of the molecule [29]. The values of distribution parameter and dielectric strength at different temperatures in SmC are listed in the Table 3. The higher values of distribution parameter suggest the existence of more than one relaxation phenomenon in the sample [30], which may be due to the typical $\lambda$ shape of the molecules of the series. The distribution parameter and dielectric strength both are almost temperature independent in $\mathrm{SmC}$ phase but a clear difference exist in the value of these parameters for $\mathrm{SmC}_{1}$ and $\mathrm{SmC}_{2}$ phases of the samples. 


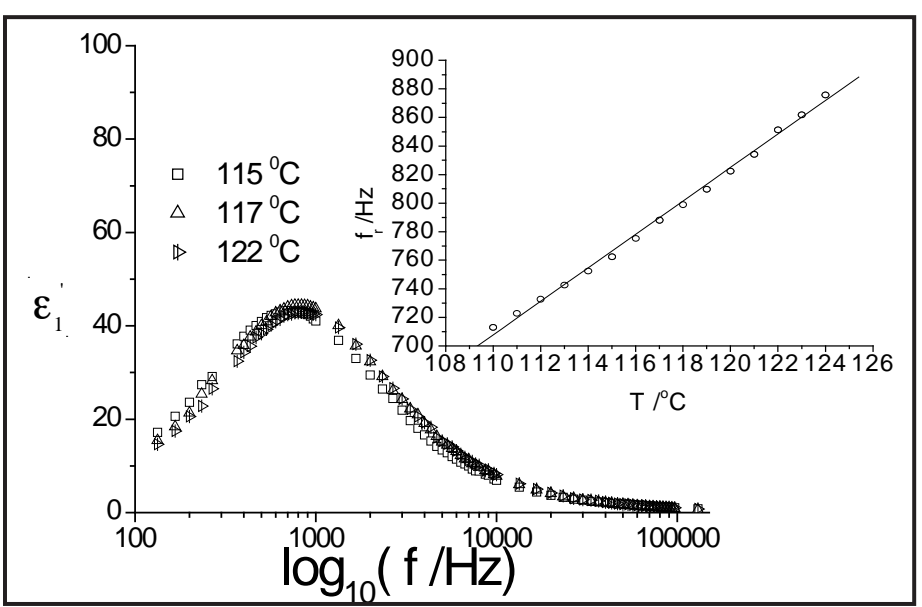

Fig. 10. The absorption curve at different temperatures and inset represents the temperature dependence of relaxation frequency of the n-octyloxy derivative in Nematic phase.

The absorption curve in nematic phase of 8th member of series is given Fig. 10. The peak in the curve indicates the relaxation frequency at any particular temperature. The absorption band in nematic phase is almost similar to the SmC phase of the element, but the temperature dependence of relaxation frequency shows an opposite behaviour as compared to SmC phase. In smectic $\mathrm{C}$ phase the relaxation frequency decreases with the increase in the temperature, while the relaxation frequency increases with increase in temperature during nematic phase (see inset Fig. 10). The higher relaxation frequency at higher temperature is quite common phenomenon and reported by many researchers [27].

\section{Conclusion}

1. A new $\lambda$-shaped mesogenic homologous series with polar bromo and nitro substituents was synthesized lower members $(n \leq 8)$ exhibit nematic mesophase except methoxy and ethoxy derivatives, which are nonmesogenic whereas higher members $(n \geq 8)$ exhibit smectic C mesophase.

2. The smectic and nematic mesophase thermal stabilities of the present series having polar nitro and bromo substituents are higher as compared to the structurally related mesogenic compounds without bromo as well as bromo and nitro substituents.

3. The samples under investigation show unusual dielectric behaviour because of peculiar $\lambda$ shape of molecules. The dielectric permittivity versus temperature curve shows the existence of two molecular arrangements in $\mathrm{SmC}$ phase.

4. The unusually dielectric permittivity values have been explained by using a model of two interconvertible conformers separated by an energy barrier, which is temperature dependent. The existence of these conformers has been confirmed by relaxation frequency versus temperature curve and by calculating the activation energies of different molecular arrangements. This has also been reflected by distribution parameters and dielectric strength as calculated by using Cole-Cole plots.

\section{Acknowledgments}

We are grateful to Dr. A. K. Prajapati, Applied Chemistry Department, Faculty of Technology and Engineering, The M. S. University of Baroda, India for providing samples.

\section{References}

A. Yamaguchi, I. Nishiyama, J. Yamamoto, H. Yokoyama and A. Yoshizawa, 2005. Unusual smectic phases organized by novel $\lambda$-shaped mesogenic molecules, J. Mater. Chem. 15, 280-288.

A. Yoshizawa and A. Yamaguchi, 2002. Kinetically induced intermolecular association: unusual enthalpy changes in the nematic phase of a novel dimeric liquid-crystalline molecule, Chem. Commun, 2060-2061.

A. Yoshizawa, M. Nakata and A. Yamaguchi, 2006. Phase transition behaviour of novel Y-shaped liquid crystal oligomers, Liq. Cryst. 33, 605-609.

A.I. Vogel, 1951. Text Book of Practical Organic Chemistry, 5th Edition, (ELBS and Longmann Group Ltd.), pp. 9461003.

A.K. Singh, R. Manohar, J.P. Shukla and A.M. Biradar, 2005/ Dielectric relaxation and anisotropy of nematic mixture E-24, J. Phys. Chem. of Solids. 66. 1183-1186.

A.K. Srivastava, A.K. Misra, P.B. Chand, R. Manohar and J.P. Shukla, 2007. Ferroelectric liquid crystals versus dyed ferroelectric liquid crystals in SmC* phase, Phys. Lett. A 371, 490-498. 
A.K. Srivastava, R. Manohar, J.P. Shukla, and A.M. Biradar, 2007. Dielectric relaxation of dye-doped ferroelectric liquid crystal mixture: a comparative study of smectic $\mathrm{C}^{*}$ and smectic A phase, Jpn. J. Appl. Phys. 46A, 1100-1105.

B.R. Ratna, R. Shashidhar and K.V. Rao, in: S. Chandrasekhar (Ed), 1980. Dielectric studies of some re-entrant nematogens, Int. Liq. Cryst. Conf., Heyden, New York, 135-142.

C. Rein and D. Demus, 1993. High-pressure differential thermal analysis of laterally substituted nematic liquid crystalline compounds, Liq. Cryst. 15, 193-202.

C.J.F. Bottcher and, P. Bordewijk, 1978. Theory of Electric Polarization, Elsevier: Amstrerdam, Vol. II, pp. 62-70.

D. Shen, D.S. Pelzl, G.I. Wirth and C. Tschierske, Designing banana-shaped liquid crystals without Schiff's base units: m-terphenyls, 2,6-diphenylpyridines and V-shaped tolane derivatives, J. Mater. Chem. 9 (1999), 661-672; H. Takezoe and Y. Takanishi, Bent-core liquid crystals: their mysterious and attractive world, Jpn. J. Appl. Phys. 45(2A) (2006), 597-625.

D. Zhang, Q. Zhou, Y. Ma, X. Wang, X. Wan and X. Feng, 1997/ Synthesis of novel liquid crystal compounds with aromatic amide mesogenic cores, Liq. Cryst. 23, 357-363.

G.W. Gray, 1962. Molecular Structure and Properties of Liquid Crystals, London and New York: Academic Press, pp. 2575.

H. Baessler, R.B. Beard and M.M. Labes, 1970. Dipole relaxation in a liquid crystal, J. Chem. Phys. 52, 2292-2298.

H. Ishizuka, I. Nishimy and A. Yoshizawa, 1995. Helical structures induced by laterally-connected chiral twin molecules, Liq. Cryst. 18, 775-779.

H. Nandedkar, Ph.D. Thesis, 1999. Synthesis and Characterization of Liquid Crystals with Flexible and Rigid Spacers, M.S. University of Baroda, Vadodara, India.

I.M. Saezand, J.W. Goodby, 2003, Design and properties of "Januslike" supermolecular liquid crystals, Chem. Commun., 1726-1727.

J. Andersch and C. Tschierske, 1996. Synthesis and liquid crystalline properties of novel laterally connected twins, Liq. Cryst. 21, 51-63.

J.S. Dave and R.A. Vora, in: J. F. Johnson and R. S. Porter (Eds), 1970. Liquid Crystals and Ordered Fluids, New York: Plenum, pp. 477-486.
J.S. Patel and J.W. Goodby, 1987. Observation of polarization sign inversion in ferroelectric liquid crystals produced by doping SC liquid crystals, J. Phys. Chem. 91, 58385840 .

J.W. Goodby, G.H. Mehl, I. M. Saez, R.P. Tuffin, G. Mackenzie, R. Auzely-Velty, T. Benvegu and D. Plusquellec, 1998. Liquid crystals with restricted molecular topologies: supermolecules and supramolecular assemblies, Chem. Commun. 2057-2070.

M. Massalska-Arodz, H. Stettin and H. Kresse, 1998. Dielectric low-frequency relaxation in a swallow-tailed liquid crystal, Mol. Cryst. Liq. Cryst. 177. 155-161.

M. Patel, Ph.D. 2000. Thesis, Synthesis and Evaluation of Liquid Crystals with Low Molecular Mass and High Molecular Mass (Polymers), M.S. University of Baroda, Vadodara, India.

N.E. Hill, W.E. Vaughan, A.H. Price and M. Davies, 1969. Dielectric Properties and Molecular Behaviour, Van Nostrand Reinhold London, pp. 1-106.

R. Dhar, A.K. Srivastava and V.K. Agrawal, 2002. Induced twist grain boundary phase in the binary mixtures of cholesteryl caprylate and nonyloxybenzoic acid, Indian J. Pure Appl. Phys. 40, 694-700.

R. Dhar, M. Gupta, V.K. Agrawal, M.K. Singh and B. Singh, 2004. Dielectric relaxation studies in-n- (4-octyloxy2-hydroxybemzylidene)-4-carbethoxyaniline, Phase Transitions 77. 375-384.

R. Mahajan, H. Nandedkarand, S. Denial. 2001. liquid crystals posessing 1,2,4-trisubstituted benzene derivatives, Mol. Cryst. Liq. Cryst. 368, 697-707.

R. Manohar, A.K. Misra, A.K. Srivastava, P.B. Chand and J.P. Shukla, 2007. Dielectric relaxation of FLC showing anomalous behavior, Soft Materials 5(4), 207-218.

R.A. Vora, A.K. Prajapati, J.B. Kevat and K.K. Raina, 2001. Mesogenic properties and the effect of 1,2,4-trisubstitution on the central benzene nucleus of a three-ring mesogen, Liq. Cryst. 28, 983-989.

S.L. Srivastava and R. Dhar, 1991. Characteristic time of ionic conductance and electrode polarization capacitance in some organic liquids by low frequency dielectric spectroscopy, Indian J. Pure \& Appl. Phys. 29, 745-751. 